Research Article

\title{
Comparative evaluation of drug susceptibility testing of Mycobacterium tuberculosis by Nitrate reductase assay on direct sputum samples and Conventional proportion method
}

\author{
Sumanta $A^{1}$, Sudhindra $\mathrm{KS}^{2}$, Nagarathnamma $\mathrm{T}^{3}$, Shubha $\mathrm{DS}^{4}$ \\ ${ }^{1}$ Dr. Sumanta A, Assistant Professor, Department of Microbiology, ${ }^{2}$ Dr. Sudhindra. K. S, Professor, Department of \\ Microbiology, both authors are affiliated with Department of Microbiology, Basaveshwara Medical College \& Hospital, \\ Chitradurga, India, ${ }^{3}$ Dr. Nagarathnamma T, Microbiology, Professor and Head (Retired), Bangalore Medical College and \\ Research Institute, Bengaluru, ${ }^{4}$ Dr. Shubha D.S, Microbiology, Professor and Head, Department of Microbiology, \\ Basaveshwara Medical College \& Hospital, Chitradurga, Karnataka, India.
}

Address for Correspondence: Dr. Sudhindra KS, Professor, Department of Microbiology, Basaveshwara Medical College \& Hospital, SJM Campus, NH-4 Bypass, Chitradurga, Karnataka. E-mail: dr_sudhindraks@yahoo.co.in

\begin{abstract}
Background and Objectives: The routinely used methods for anti-tubercular drug susceptibility testing are either costly or slow. As the prevalence of multidrug-resistant strains is increasing, the need for fast, reliable, and inexpensive methods that can be applied in settings with limited resources is essential. Methods: It was a study of 100 sputum samples from smear positive patients at RNTCP centre. The samples were subjected to anti-tubercular drug susceptibility by Nitrate reductase assay on direct sputum samples and the routine indirect Conventional proportion method for two primary anti-tubercular drugs, i.e., Isoniazid, and Rifampicin. Results: Out of 100 samples, 94 were sensitive to Isoniazid and Rifampicin by both Conventional Proportion Method, and Nitrate Reductase Assay. Four isolates were detected as MDR-TB strains (resistant to both Isoniazid and Rifampicin) and two were resistant only to Isoniazid by both the methods. Conclusion: Drug susceptibility detected by Nitrate Reductase Assay has excellent agreement with the gold standard Conventional proportion method for Mycobacterium tuberculosis in our study. Hence in countries like India, where there is burden of tuberculosis cases and especially of drug resistant cases, NRA is a very valuable tool in the detection, treatment and follow up of tuberculosis cases for drug resistance.
\end{abstract}

Keywords: Tuberculosis (TB), Multi drug resistant tuberculosis (MDR-TB), Nitrate reductase assay (NRA), Conventional Proportion Method (CPM)

\section{Introduction}

Tuberculosis (TB) has been one of the fatal human infections with many serious consequences, affecting humans since antiquity. The situation has been worsened due to the emergence of multi drug resistant tuberculosis (MDR-TB), extensively drug resistant tuberculosis (XDR-TB) and HIV-TB co-infection [1]. The rapid identification of multi drug resistant isolates is essential for the effective treatment of patients. Conventional drug susceptibility test results become available only after 4 to 6 weeks after isolation by culture. Culture itself takes additional 4 to 8 weeks.

Manuscript received: $15^{\text {th }}$ May 2017

Reviewed: 26 ${ }^{\text {th }}$ May 2017

Author Corrected: $5^{\text {th }}$ June 2017

Accepted for Publication: 14 $4^{\text {th }}$ June 2017
Liquid culture methods like BACTEC MGIT 960 take much lesser time but the initial infrastructure requirement and the cost per test are a bit high to afford in many laboratories across India. An efficient and rapid drug susceptibility testing becomes necessary in such a situation.

The nitrate reductase assay (NRA), is a simple and less expensive technique based on the characteristic ability of $M$. tuberculosis to reduce nitrate to nitrite. Hence, in this study we tried to study the efficacy of Nitrate reductase assay as an alternative to the Conventional proportion method (CPM) for anti-tubercular drug susceptibility testing. 


\section{Materials and Methods}

Sputum samples from smear positive patients visiting our RNTCP centre were collected in a sterile wide mouthed container. The collected samples were subjected to decontamination and concentration by modified Petroff's method [2]. Biosafety class II cabinet was used for procedure.

Smear-positive sputum samples (Positive for AFB with $1+, 2+$ or $3+$ grade) were inoculated directly for NRA [3] after decontamination and concentration. For NRA, the sediment was suspended in $3 \mathrm{ml}$ of sterile distilled water and $200 \mu \mathrm{l}$ was inoculated onto LowensteinJenson (L-J) medium with $1,000 \mu \mathrm{g} / \mathrm{ml}$ of potassium nitrate with antibiotics, i.e., $0.2 \mu \mathrm{g} / \mathrm{ml}$ for Isoniazid (INH), and $40 \mu \mathrm{g} / \mathrm{ml}$ for Rifampicin (RIF) separately. The control for this test was an L-J slant with 1,000 $\mu \mathrm{g} / \mathrm{ml}$ of potassium nitrate without any antibiotics. A total of three of these control media were inoculated at 1:10 dilution of the above mentioned sediment. All tubes were incubated at $37^{\circ} \mathrm{C}$. NRA was based on ability of Mycobacterium tuberculosis to reduce nitrate to nitrite, which was revealed by color change in the medium after addition of Griess reagent [3]. The results were classified as negative (sensitive) if no color change occurred in the antibiotic containing media and positive (resistant) if there was pink to red color development. Color intensity was compared with that of the control. After 7 days of incubation, Griess reagent was added to one of the control tubes. If found positive (pink to red color), it was then tested for the antibioticcontaining tubes. Any change of color (more than the control tube) was taken as resistance. If the control tube did not show any color change after addition of Griess reagent, other tubes were further incubated and the procedure was repeated at 14 days and 21 days. The results of the NRA were compared with the CPM. The CPM was performed using L-J medium according to the standard procedure [4] with the recommended critical concentration of INH, and RIF. H37Rv served as a standard control for both the tests.

\section{Results}

A total of 100 sputum samples with smear positivity were subjected to both direct NRA (without isolation of Mycobacterium tuberculosis) and indirect CPM (after isolation M. tuberculosis) and compared. Out of 100 samples, 94 were sensitive to both Isoniazid and Rifampicin by both Conventional Proportion Method, and Nitrate Reductase Assay (Table 1). Four isolates were detected as MDR-TB strains, i.e., resistant to both Isoniazid and Rifampicin, and two were resistant only to Isoniazid by both the methods. The results showed that NRA and CPM do not differ for both drugs. Thus an excellent agreement between the results of NRA and CPM was found for Isoniazid and Rifampicin of $100 \%$. In our study, results were available in 7 days for 42 strains, 14 days for 39 strains, in 21 days for 19 strains. When compared to the CPM, the results of NRA were available two to three weeks ahead of CPM. Also another added advantage is that there is no need to isolate the organism by culture as the drug-susceptibility by NRA was done on direct sputum samples, thereby further reducing the time.

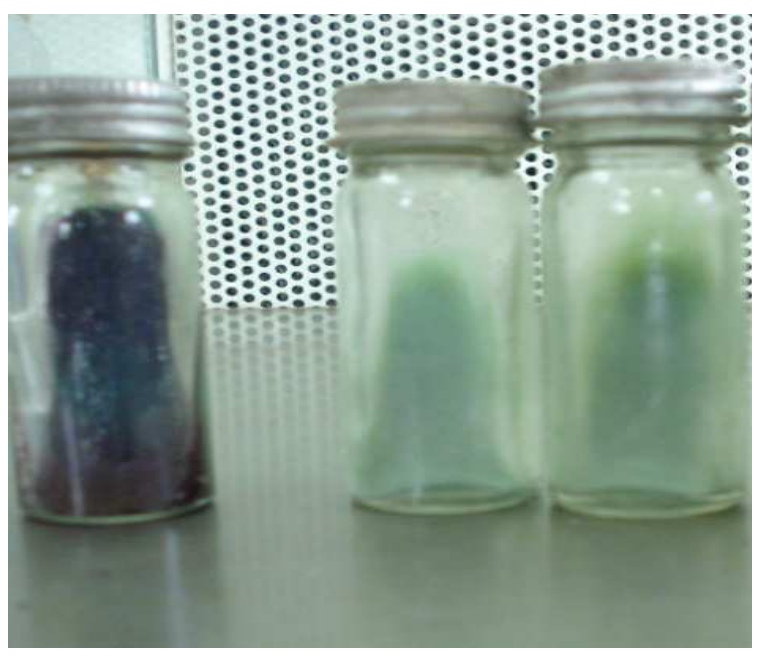

Fig.-1: Nitrate Reductase Assay - sensitive isolate (no colour change obtained on LJ medium with anti tubercular drugs - INH, RIF in this order from left to right). Extreme left - control tube without anti tubercular drugs. 


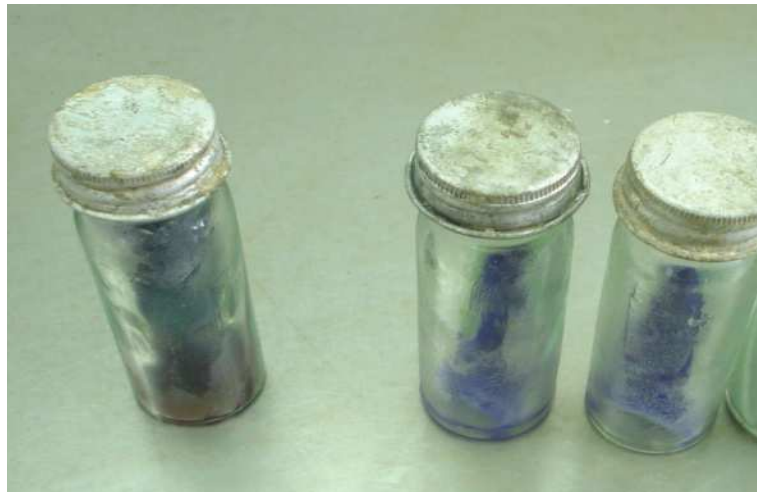

Fig.-2: Nitrate Reductase Assay- MDR isolate. Dark purple colour change is present on all tubes. (INH, RIF in this order from left to right). Extreme left - control tube without anti tubercular drugs.

Table-1: Drug susceptibility to INH, RIF

\begin{tabular}{|c|c|}
\hline Sensitive or resistant to particular drug & Number of cases \\
\hline sensitive to Isoniazid & 94 \\
\hline resistant to Isoniazid & 06 \\
\hline sensitive to Rifampicin & 96 \\
\hline resistant to Rifampicin & 04 \\
\hline
\end{tabular}

The same result is obtained by both the methods, i.e., conventional proportion method and nitrate reductase assay.

\section{Discussion}

NRA is simple to perform and does not require additional equipment and reagents than those used for Conventional Proportion Method. NRA is performed on classical LJ media used routinely in all TB laboratories. Results are simple to interpret by change of color. Also, biosafety problems are limited as the test is performed in a solid medium reducing the risk of production of aerosols during work.

Sensitivities and specificities of direct method of Nitrate reductase assay are $100 \%$ for both the drugs, i.e., INH, and RIF. Out of the 100 isolates, 94 isolates were sensitive for both INH, and RIF. 04 isolates were multidrug resistant (MDR). The same result was obtained by Conventional proportion method which is done on the isolates obtained on LJ media (Indirect method). There was $100 \%$ agreement between Conventional proportion method and Nitrate reductase assay for drug susceptibility testing of both INH and Rifampicin from our study.

With reference to NRA, Mishra et al [3] have shown agreement of $87.5 \%$ for INH and $97 \%$ for Rifampicin. Sethi et al [5] have shown 100\% agreement for Rifampicin and $99 \%$ for INH. Musa et al [6] have shown $93 \%$ and $100 \%$ agreement for INH and Rifampicin respectively. Similar findings were also observed by other authors $[7,8,9,10,11$, and 12]. The findings were analyzed and supported in the metaanalysis by Martin et al [13], in which they have mentioned the need for more such studies in countries with high prevalence of tuberculosis.

When compared to the CPM, the results of direct NRA were available two to three weeks ahead of CPM with an added advantage of no necessity to isolate the organism. Liquid culture methods like BACTEC MGIT 960 take much lesser time but the initial infrastructure requirement and the cost per test are a bit high to afford in many laboratories across India. Nitrate reductase negative strains of $M$. tuberculosis are rare $(<1 \%)$ and would create no false results since the control would be negative and the test would therefore be invalid [7]. No such strains were encountered in our study.

\section{Conclusion}

NRA is simple to perform, and provides a rapid, accurate, and cost effective means for the detection of Isoniazid and Rifampicin resistance in M. tuberculosis. Drug susceptibility detected by direct Nitrate Reductase Assay (NRA) has excellent agreement with the gold standard Conventional proportion method for $M$. tuberculosis. NRA is also a phenotypic method as 
Conventional proportion method, which produces standard results. The newer genotypic methods such as Line Probe Assay and GeneXpert won't identify all the genes responsible for resistance whereas phenotypic methods do. Hence in countries like India, where there is burden of tuberculosis cases (especially of drug resistant cases), NRA is a very valuable tool in the detection, treatment and follow up of tuberculosis cases for drug resistance.

Funding: Nil, Conflict of interest: None initiated, Permission from IRB: Yes

\section{References}

1. Fitzgerald D, Haas DW. Mycobacterium, Chapter 248. In: Mandell GL, Bennett JE and Dolin R Principles and practice of infectious diseases. $6^{\text {th }}$ Edition. (Elsevier, Churchill Livingstone); 2005. P.2852-83.

2. Allen BW, Baker FJ. Modified Petroff's Technique Treatment of specimens, Chapter 3 in Mycobacteria Isolation, Identification and Sensitivity Testing. Allen BW, Baker FJ. Butterworth \& Co. London. 1968; 9-16

3. Mishra B, Muralidharan S, Srinivasa H. Direct drug susceptibility testing of Mycobacterium tuberculosis to primary anti-tubercular drugs by nitrate reductase assay. Indian J Pathol Microbiol 2009; 52(3):343-4.

4. RNTCP training manual for Mycobacterium tuberculosis culture and drug susceptibility testing. April 2009. Central TB division. Directorate General of Health Services. New Delhi.

5. Sethi S, Sharma S, Sharma SK, Meharwal SK, Jindal SK and Sharma M. Drug susceptibility of Mycobacterium tuberculosis to primary antitubercular drugs by nitrate reductase assay. Indian J Med Res 2004; 120 (5):468-71.

6. Musa HR, Ambroggi M, Souto A, Angeby KAK. Drug susceptibility testing of Mycobacterium tuberculosis by a Nitrate Reductase Assay applied directly on microscopy positive sputum samples. J Clin Microbiol 2005;43(7): 3159-61

7. Angeby KAK, Klintz L, Hoffner SE. Rapid and Inexpensive drug susceptibility testing of Mycobacterium tuberculosis with nitrate reductase assay. J Clin Microbiol 2002; 40(2):553-5.

8. Ani AE, Dalyop YB, Agbaji O, Idko J. Drug susceptibility test of Mycobacterium tuberculosis by nitrate reductase assay. J Infect Dev ctries 2009; 3(1): 16-9.

9. Coban AY, Birinci A, Ekinci B, Durupinar B. Drug susceptibility testing of Mycobacterium tuberculosis with nitrate reductase assay. Int $\mathbf{J}$ Antimicrob Agents 2004; 24(3):304-6.

10. Shikama ML, Silva RE, Martins MC, Giampagelia CMS, Oliveira RS, Silva RFM et al. Rapid detection of resistant tuberculosis by nitrate reductase assay performed in three settings in Brazil. J Antimicrob Chemother 2009; 64(4):794-6.

11. Lemus D, Montoro E, Echemendia M, Martin A, Portaels F, Palomino JC. Nitrate reductase assay for detection of drug resistance in Mycobacterium tuberculosis: Simple and inexpensive method for lowresource laboratories. J Med Microbiol 2006; 55(7): 861-3.

12. Affolabi D, Odoun M, Martin A, Palomino JC, Anagonou S, Portaels F. Evaluation of direct detection of Mycobacterium tuberculosis Rifampicin resistance by nitrate reductase assay applied to sputum samples in Cotonou, Benin. J Clin Microbiol 2007; 45(7):2123-5

13. Martin A, Panaiotov S, Portails F, Hoffner S, Palomino JC and Angeby K. The nitrate reductase assay for the rapid detection of isoniazid and rifampicin resistance in Mycobacterium tuberculosis: a systemic review and meta-analysis. J Antimicrob chemother 2008;62(1):56-64.

\section{How to cite this article?}

Sumanta A, Sudhindra KS, Nagarathnamma T, Shubha DS. Comparative evaluation of drug susceptibility testing of Mycobacterium tuberculosis by Nitrate reductase assay on direct sputum samples and Conventional proportion method. Trop J Path Micro 2017;3(2):219-222.doi: 10.17511/jopm.2017.i2.25. 